\title{
Identification of key genes in colorectal cancer using random walk with restart
}

\author{
XIAOFENG CUI $^{1}$, KEXIN SHEN $^{1}$, ZHONGSHI XIE $^{1}$, TONGJUN LIU ${ }^{2}$ and HAISHAN ZHANG ${ }^{1}$ \\ ${ }^{1}$ Department of Gastrointestinal Colorectal and Anal Surgery, China-Japan Union Hospital, Jilin University, \\ Changchun, Jilin 130033; ${ }^{2}$ Department of General Surgery, Jilin University, Changchun, Jilin 130022, P.R. China
}

Received November 18, 2015; Accepted November 15, 2016

DOI: $10.3892 / \mathrm{mmr} .2016 .6058$

\begin{abstract}
As the most common type of cancer and the second leading cause of cancer-associated mortality, colorectal cancer (CRC) has received increasing attention. The aim of the present study was to investigate the mechanisms of CRC by analyzing the microarray dataset, GSE32323. The GSE32323 dataset was downloaded from the Gene Expression Omnibus, and included 17 pairs of matched cancer and normal colorectal tissue samples. The differentially expressed genes (DEGs) were screened using the Linear Models for Microarray Data package and a search of CRC genes, also denoted as seed genes, was performed using the Online Mendelian Inheritance in Man database. Subsequently, the protein-protein interaction (PPI) network was downloaded from the Search Tool for the Retrieval of Interacting Genes database and the sub-network (CRC.PPI) of the DEGs and seed genes were obtained. In addition, the top 50 nodes with highest affinity scores in the CRC.PPI were identified using random walk with restart analysis. The potential functions of the DEGs included in the top 50 nodes were analyzed using the Database for Annotation, Visualization and Integrated Discovery online tool. Using the Drug Gene Interaction database, drug-gene interaction analysis was performed to identify antineoplastic drug interacts with genes. A total of 1,640 DEGs between the CRC and normal samples were screened. The obtained seed genes included cyclin D1 (CCNDI) and aurora kinase A (AURKA). The enriched functions for the 31 DEGs in the PPI
\end{abstract}

Correspondence to: Dr Tongjun Liu, Department of General Surgery, Jilin University, 218 Ziqiang Street, Changchun, Jilin 130022, P.R. China

E-mail: tongjunliu@163.com

Dr Haishan Zhang, Department of Gastrointestinal Colorectal and Anal Surgery, China-Japan Union Hospital, Jilin University, 126 Xiantai Street, Changchun, Jilin 130033, P.R. China

E-mail: haishanzh@163.com

Key words: colorectal cancer, differentially expressed genes, seed genes, protein-protein interaction network, drug-gene interaction analysis network of the top 50 nodes were predominantly associated with cell cycle. The DEGs may function in CRC by interacting with other genes in the PPI network of the top 50 nodes, for example, DEP domain-containing MTOR-interacting protein (DEPTOR)-CCND1, AURKA-breast carcinoma amplified sequence-1 (BCAS1), CCND1-BCAS1, CCND1-neural precursor cell expressed developmentally downregulated 9 (NEDD9) and CCND1-mitogen-activated protein kinase kinase 2 (MAP2K2). Only three DEGs (CCND1, AURKA and DEPTOR) had interactions with their corresponding antineoplastic drugs. Taken together, DEPTOR, AURKA, CCNDI, $B C A S 1, N E D D 9$ and $M A P 2 K 2$ may act in CRC.

\section{Introduction}

As a type cancer occurring in the rectum or colon, colorectal cancer (CRC) is also termed rectal cancer or colon cancer (1). Patients with CRC often have symptoms of persistent tiredness, altered bowel movements, blood in stools and weight loss (2). With a mortality rate of $\geq 200,000$ per year in Europe (3), CRC is the most common type of cancer and ranks as at second leading cause of cancer-associated mortality (4).

Several studies have been performed to investigate the effects of genes on CRC. For example, cyclooxygenase-2, which is mediated by vascular endothelial growth factor, can function in the tumor angiogenesis of CRC (5). In mutations of the catalytic subunit $\alpha$ of phosphatidylinositol-3-kinase (PIK3CA), all mutations are functionally active in colon cancer, therefore, it may be associated with carcinogenesis (6). Mutations in Kirsten-ras, adenomatous polyposis coli and $p 53$ induce the transition from healthy colonic epithelia to CRC (7). The epigenetic loss of function of secreted frizzled-related protein may provide constitutive WNT signaling, which is essential for downstream mutations of complement in the progression of CRC (8). As an epigenetic alteration, loss of imprinting can affect the insulin-like growth factor II gene and may be a useful predictor for the risk of CRC (9).

In 2012, Khamas et al (10) performed oligonucleotide microarray analysis to identify the differentially expressed genes (DEGs) between CRC samples and normal samples. Using the same data as that used by Khamas et al (10), the present study aimed to further identify the DEGs and CRC genes. A sub-network of the DEGs and seed genes were obtained from the downloaded protein-protein interaction 
(PPI) network, termed the CRC.PPI. In addition, random walk with restart (RWR) analysis was performed to identify the 50 nodes with the top affinity scores in the CRC.PPI. The potential functions of the DEGs included in the 50 key nodes were then analyzed using Gene Ontology (GO) and pathway enrichment analyses. In addition, drug-gene interaction analysis was performed to identify interactions between antineoplastic drugs and genes.

\section{Materials and methods}

Microarray data. The expression profile of GSE32323, deposited by Khamas et al (10) was downloaded from the Gene Expression Omnibus (www.ncbi.nlm.nih.gov/geo/), which was based on the platform of the GPL570 (HG-U133_Plus_2) Affymetrix Human Genome U133 Plus 2.0 array (Affymetrix, Inc., Santa Clara, CA, USA). The GSE32323 profile included a collection of 17 pairs of matched cancer-normal colorectal tissue samples. The CRC samples were obtained from the American Type Culture Collection (Manassas, VA, USA) and the Cell Resource Center for Biomedical Research of Tohoku University (Sendai, Japan). The cells were cultured in media obtained from Sigma-Aldrich; Merck Millipore (Darmstadt, Germany) or Gibco; Thermo Fisher Scientific, Inc. (Waltham, MA, USA), supplemented with $100 \mu \mathrm{g} / \mathrm{ml}$ streptomycin (Invitrogen; Thermo Fisher Scientific, Inc.), $100 \mathrm{U} / \mathrm{ml}$ penicillin and $10 \%$ heat-inactivated fetal bovine serum (Nichirei Biosciences, Tokyo, Japan).

Identification of DEGs and CRC genes. Following downloading of the GSE32323 profile, the microarray data were preprocessed using the Affy package (11) in Bioconductor (www.ncbi.nlm.nih.gov/geo/) and the Affy probe annotation file from Brain Array Lab (brainarray.mhri.med.umich. edu/brainarray/default.asp). In brief, the process included background correction, quantile normalization, probe summarization, and $\log 2$ conversion. The mean values of probes mapped with the same gene were obtained as ultimate gene expression values. Subsequently, the Linear Models for Microarray Data (Limma) package in R (www.bioconductor. org/packages/release/bioc/html/limma.html) (12) was used to screen the DEGs of the CRC samples, compared with the normal samples. An adjusted $\mathrm{P}$-value of $\mathrm{P}<0.05$ and |logfold-changel $>1$ were used as the cut-off criteria. The CRC genes were identified from a search using the Online Mendelian Inheritance in Man (OMIM) database (www.ncbi. nlm.nih.gov/omim) (13) and were then annotated as seed genes.

PPInetwork construction and RWR analysis. The PPI network was downloaded from the Search Tool for the Retrieval of Interacting Genes database (string-db.org/) (14). The sub-network of the DEGs and seed genes was then obtained and termed the CRC.PPI. The RWR algorithm was originally used for image segmentation (15). With a restarting probability (r) of 0.9 , the R package dnet (16) was used to perform RWR analysis to identify the top 50 nodes with the highest affinity scores in the CRC.PPI. The affinity score referred to the proximity between two nodes and the parameter ' $r$ ' is the probability of moving to seed nodes.
Functional and pathway enrichment analysis. The Database for Annotation, Visualization and Integrated Discovery (DAVID; david.abcc.ncifcrf.gov/) is a tool for agglomerating a large quantity of gene/protein symbols into gene clusters (17). Gene Ontology (GO) analysis is used to predict the potential functions of large-scale transcriptomic data or genomic data (18). The Kyoto Encyclopedia of Genes and Genomes database is a base for functional analysis combing with networks of genes and other molecules (19). Using the DAVID online tool, GO functional and pathway enrichment analyses were performed for the DEGs in the PPI network of the top 50 nodes. $\mathrm{P}<0.05$ was used as the cut-off criterion.

Drug-gene interaction analysis. The Drug-Gene Interaction database (dgidb.genome.wustl.edu/) (20) was used to identify interactions between antineoplastic drugs and genes. The actions of the drugs inhibit genes expressed at high levels and promote genes expressed at low levels.

\section{Results and Discussion}

DEG analysis. Compared with the normal samples, there were 1,640 DEGs screened in the CRC samples, including 850 upregulated genes and 790 downregulated genes. The CRC genes or seed genes from the OMIM database are listed in Table I, including cyclin D1 (CCNDI) and aurora kinase A (AURKA).

PPI network analysis. Following downloading of the PPI network and obtaining the CRC.PPI of the DEGs and seed genes, RWR analysis was performed and nodes with the top 50 affinity scores, which included DEP domain-containing MTOR-interacting protein (DEPTOR), breast carcinoma amplified sequence-1 (BCAS1), neural precursor cell expressed developmentally downregulated 9 (NEDD9), mitogen-activated protein kinase kinase 2, (MAP2K2) in the CRC.PPI were identified. The PPI network of the top 50 nodes with the highest affinity scores, which comprised 14 upregulated genes, 17 downregulated genes and 19 non-DEGs, had 224 interactions. These interactions included DEPTOR-CCND1, AURKA-BCAS1, CCND1-BCAS1, CCND1-NEDD9 and CCND1-MAP2K2 (Fig. 1). The DEGs included in the top 50 nodes are listed in Table II. The enriched functions of the 31 DEGs in the PPI network of the top 50 nodes were primarily associated with cell cycle, including cell cycle phase $(\mathrm{P}=0.005651), \mathrm{M}$ phase $(\mathrm{P}=0.019763)$ and intracellular signaling cascade $(\mathrm{P}=0.019784)$, as shown in Table III. The enriched pathway for the 31 DEGs in the PPI network of the top 50 nodes included cytokine-cytokine receptor interaction $(\mathrm{P}=0.04602$; Table III).

Drug-gene interaction analysis. The interactions between antineoplastic drugs and the 31 DEGs in the CRC.PPI network were screened. Only three DEGs (CCND1, AURKA and $D E P T O R$ ), had interactions with their corresponding antineoplastic drugs.

For the analysis of drug-gene interactions in the present study, a total of 1,640 DEGs were screened in the CRC samples, compared with normal samples, which included 850 upregulated genes and 790 downregulated genes. The 
Table I. Seed genes from an Online Mendelian Inheritance in Man database search.

\begin{tabular}{|c|c|c|}
\hline Gene/locus & ID & Name \\
\hline$P L A 2 G 2 A$ & 5320 & Phospholipase A2, group IIA (platelets, synovial fluid) \\
\hline$N R A S$ & 4893 & Neuroblastoma RAS viral (v-ras) oncogene homolog \\
\hline$O D C 1$ & 4953 & Ornithine decarboxylase 1 \\
\hline CTNNB1 & 1499 & Catenin (cadherin-associated protein), $\beta 1,88 \mathrm{kDa}$ \\
\hline$P I K 3 C A$ & 5290 & Phosphatidylinositol-4,5-bisphosphate 3-kinase, catalytic subunit $\alpha$ \\
\hline FGFR3 & 2261 & Fibroblast growth factor receptor 3 \\
\hline$T L R 2$ & 7097 & Roll-like receptor 2 \\
\hline$A P C$ & 324 & Adenomatous polyposis coli \\
\hline$M C C$ & 4163 & Mutated in colorectal cancers \\
\hline PTPN12 & 5782 & Protein tyrosine phosphatase, non-receptor type 12 \\
\hline PDGFRL & 5157 & Platelet-derived growth factor receptor-like \\
\hline$R A D 54 B$ & 25788 & RAD54 homolog B (S. cerevisiae) \\
\hline TLR4 & 7099 & Toll-like receptor 4 \\
\hline PTPRJ & 5795 & Protein tyrosine phosphatase, receptor type, $\mathrm{J}$ \\
\hline CCND1 & 595 & Cyclin D1 \\
\hline MLH3 & 27030 & MutL homolog 3 \\
\hline$A K T 1$ & 207 & V-akt murine thymoma viral oncogene homolog 1 \\
\hline$B U B 1 B$ & 701 & BUB1 mitotic checkpoint serine/threonine kinase B \\
\hline TP53 & 7157 & Tumor protein p53 \\
\hline FLCN & 201163 & Folliculin \\
\hline AXIN2 & 8313 & Axin 2 \\
\hline$D C C$ & 1630 & Deleted in colorectal carcinoma \\
\hline$B A X$ & 581 & BCL2-associated X protein \\
\hline$A U R K A$ & 6790 & Aurora kinase A \\
\hline EP300 & 2033 & E1A binding protein p300 \\
\hline
\end{tabular}

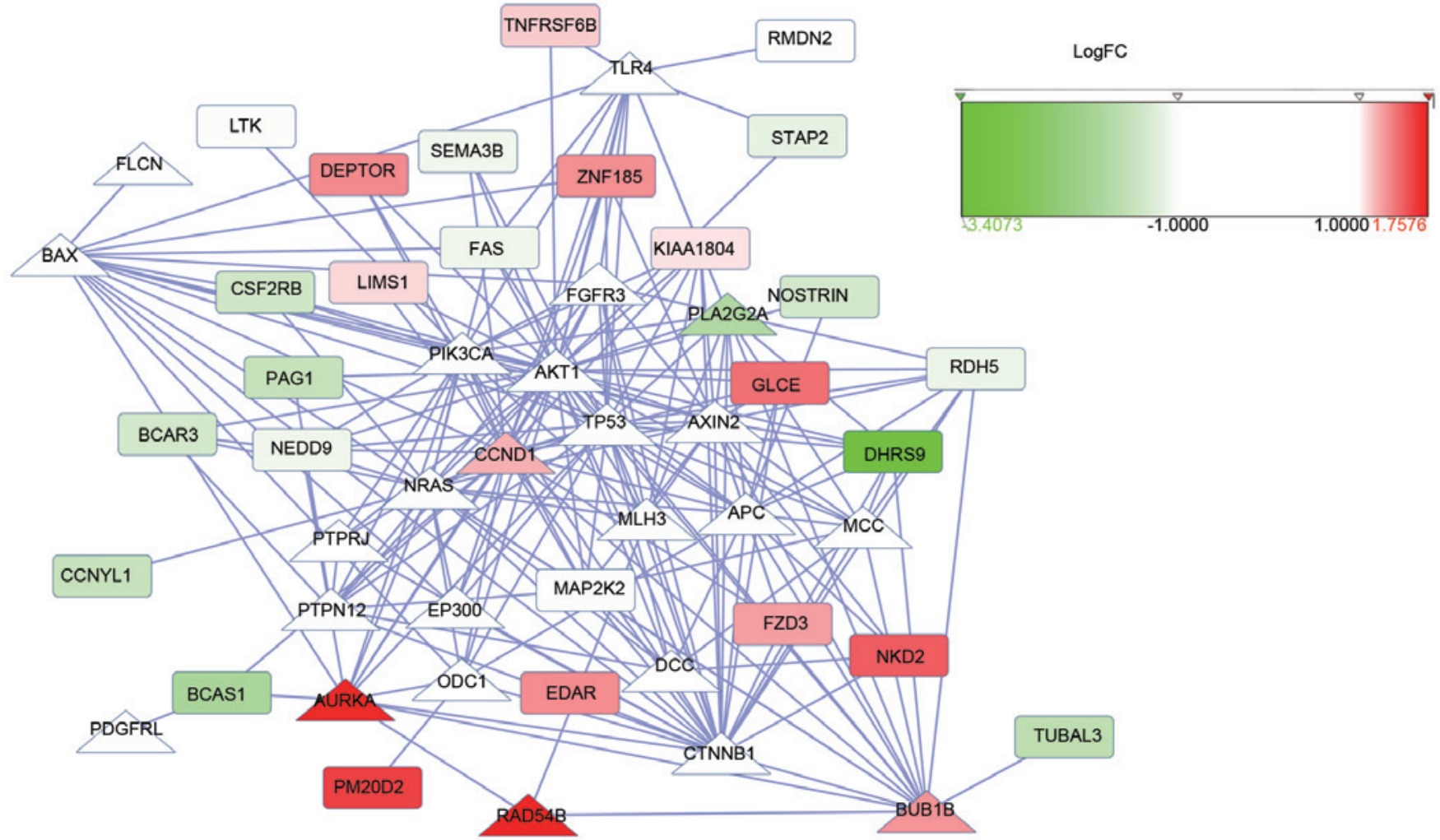

Figure 1. Protein-protein interaction network of the top 50 nodes with the highest affinity scores. 
Table II. 31 differentially expressed genes in the protein-protein interaction network of the top 50 nodes.

\begin{tabular}{|c|c|c|c|c|}
\hline Gene & Seed & Affinity score & $\log \mathrm{FC}$ & P-value \\
\hline$P L A 2 G 2 A$ & $\mathrm{Y}$ & 0.033061 & -1.790530 & $2.46 \mathrm{E}-02$ \\
\hline$C C N D 1$ & $\mathrm{Y}$ & 0.032774 & 1.195653 & $5.43 \mathrm{E}-06$ \\
\hline$B U B 1 B$ & Y & 0.032755 & 1.290377 & $3.10 \mathrm{E}-03$ \\
\hline AURKA & $\mathrm{Y}$ & 0.032712 & 1.748420 & $3.98 \mathrm{E}-05$ \\
\hline$R A D 54 B$ & $\mathrm{Y}$ & 0.032643 & 1.757562 & $1.64 \mathrm{E}-08$ \\
\hline LTK & $\mathrm{N}$ & 0.001651 & -1.023600 & $1.24 \mathrm{E}-05$ \\
\hline RDH5 & $\mathrm{N}$ & 0.001267 & -1.146630 & $1.34 \mathrm{E}-08$ \\
\hline NOSTRIN & $\mathrm{N}$ & 0.001110 & -1.379460 & $1.42 \mathrm{E}-04$ \\
\hline DHRS9 & $\mathrm{N}$ & 0.001109 & -3.407330 & $9.77 \mathrm{E}-05$ \\
\hline$R M D N 2$ & $\mathrm{~N}$ & 0.001097 & -1.010210 & $7.66 \mathrm{E}-04$ \\
\hline$P A G 1$ & $\mathrm{~N}$ & 0.000960 & -1.535700 & $2.55 \mathrm{E}-05$ \\
\hline SEMA3B & $\mathrm{N}$ & 0.000917 & -1.108180 & 7.87E-07 \\
\hline$B C A S 1$ & $\mathrm{~N}$ & 0.000903 & -1.881610 & $1.78 \mathrm{E}-06$ \\
\hline$M A P 2 K 2$ & $\mathrm{~N}$ & 0.000881 & -1.020110 & $3.50 \mathrm{E}-07$ \\
\hline$C S F 2 R B$ & $\mathrm{~N}$ & 0.000838 & -1.414110 & $8.49 \mathrm{E}-05$ \\
\hline TUBAL3 & $\mathrm{N}$ & 0.000837 & -1.631620 & $2.32 \mathrm{E}-03$ \\
\hline STAP2 & $\mathrm{N}$ & 0.000829 & -1.175130 & $5.35 \mathrm{E}-03$ \\
\hline CCNYLI & $\mathrm{N}$ & 0.000828 & -1.516030 & $3.86 \mathrm{E}-06$ \\
\hline NEDD9 & $\mathrm{N}$ & 0.000808 & -1.110480 & $6.60 \mathrm{E}-05$ \\
\hline BCAR3 & $\mathrm{N}$ & 0.000777 & -1.380050 & $8.69 \mathrm{E}-09$ \\
\hline$F A S$ & $\mathrm{~N}$ & 0.000768 & -1.110550 & $1.40 \mathrm{E}-04$ \\
\hline KIAA1804 & $\mathrm{N}$ & 0.001365 & 1.058162 & $2.43 \mathrm{E}-03$ \\
\hline$E D A R$ & $\mathrm{~N}$ & 0.001124 & 1.319916 & $2.78 \mathrm{E}-04$ \\
\hline DEPTOR & $\mathrm{N}$ & 0.001112 & 1.325986 & 4.94E-05 \\
\hline LIMS1 & $\mathrm{N}$ & 0.000946 & 1.088253 & 7.41E-09 \\
\hline ZNF185 & $\mathrm{N}$ & 0.000912 & 1.31012 & $2.84 \mathrm{E}-06$ \\
\hline$N K D 2$ & $\mathrm{~N}$ & 0.000910 & 1.495638 & 4.17E-08 \\
\hline FZD3 & $\mathrm{N}$ & 0.000898 & 1.25656 & $2.96 \mathrm{E}-05$ \\
\hline GLCE & $\mathrm{N}$ & 0.000843 & 1.419823 & 7.12E-06 \\
\hline TNFRSF6B & $\mathrm{N}$ & 0.000829 & 1.121187 & $3.31 \mathrm{E}-03$ \\
\hline PM20D2 & $\mathrm{N}$ & 0.000822 & 1.602442 & $1.00 \mathrm{E}-06$ \\
\hline
\end{tabular}

FC, fold-change; Y, yes; N, no.

obtained seed genes included CCNDI and AURKA. The enriched functions for the 31 DEGs in the PPI network of the top 50 nodes were primarily associated with cell cycle. According to the drug-gene interaction analysis, only three DEGs (CCNDI, AURKA and DEPTOR) had interactions with their corresponding antineoplastic drugs.

DEPTOR, which is negatively correlated with tumor progression and the activity of mammalian target of rapamycin complex 1 (mTORCl) in CRC, may function as a marker for prognosis and treatment of CRC (21). The PI3K/Akt/mTOR signaling pathway is one of the primary mechanisms involved in maintaining tumor progression and metastasis, and mTOR activation/inhibition functions in CRC (22). It has been reported that DEPTOR may be closely correlated with CRC. The variants in $C C N D 1$ may have potential associations with familial CRC (23), and the CCND1 A870G polymorphism may increase the risk of CRC (24). The epidermal growth factor A61G and CCND1 A870G polymorphisms may be valuable molecular biomarkers for the prognosis of patients with CRC treated with the single-agent, cetuximab (25). Thus, the expression levels of CCND1 may be associated with CRC. The PPI network of the top 50 nodes in the present study also showed CCND1 had an interaction with DEPTOR, indicating that CCND1 may be involved in CRC by mediating DEPTOR.

Independent of established clinicopathological variables, AURKA can act as a molecular marker for predicting clinical outcome in patients with liver metastasis of CRC (26). AURKA, which is located on chromosome $20 \mathrm{q}$, is involved in adenoma-to-carcinoma progression and may function as an indicator of poor prognosis (27-33). $N A B C 1$, also known as $B C A S 1$, and its variant, $N A B C 15 B$, are downregulated in CRC (34). These findings suggest that AURKA and BCASI may be associated with CRC. In the PPI network of the top 50 nodes in the present study, it was also found that BCAS1 interacted with AURKA, suggesting that BCAS1 may also be involved in CRC through regulating AURKA. 
Table III. Enriched functions and pathways for the 31 differentially expressed genes in the protein-protein interaction network of the top 50 nodes.

\begin{tabular}{|c|c|c|c|c|c|}
\hline Category & ID & Description & Genes (n) & Gene symbol & P-value \\
\hline GO & 0022403 & Cell cycle phase & 5 & $\begin{array}{l}\text { CCND1, BUB1B, NEDD9, } \\
\text { AURKA, RAD54B }\end{array}$ & 0.005651 \\
\hline GO & 0022402 & Cell cycle process & 5 & $\begin{array}{l}\text { CCND1, BUB1B, NEDD9, } \\
\text { AURKA, RAD54B }\end{array}$ & 0.016449 \\
\hline GO & 0000279 & M phase & 4 & $\begin{array}{l}\text { BUB1B, NEDD9, AURKA, } \\
\text { RAD54B }\end{array}$ & 0.019763 \\
\hline GO & 0007242 & Intracellular signaling cascade & 7 & $\begin{array}{l}\text { CCND1, MAP2K2, KIAA1804, } \\
\text { BUB1B, AURKA, PAG1, BCAR3 }\end{array}$ & 0.019784 \\
\hline GO & 0016055 & Wnt receptor signaling pathway & 3 & CCND1, NKD2, FZD3 & 0.022988 \\
\hline GO & 0000278 & Mitotic cell cycle & 4 & CCND1, BUB1B, NEDD9, AURKA & 0.026838 \\
\hline GO & 0006468 & Protein amino acid phosphorylation & 5 & $\begin{array}{l}\text { CCND1, LTK, MAP2K2, } \\
\text { KIAA1804, AURKA }\end{array}$ & 0.028349 \\
\hline GO & 0048545 & Response to steroid hormone stimulus & 3 & CCND1, AURKA, FAS & 0.045081 \\
\hline GO & 0007049 & Cell cycle & 5 & $\begin{array}{l}\text { CCND1, BUB1B, NEDD9, } \\
\text { AURKA, RAD54B }\end{array}$ & 0.045692 \\
\hline KEGG & 04060 & Cytokine-cytokine receptor interaction & 4 & TNFRSF6B, CSF2RB, EDAR, FAS & 0.046018 \\
\hline
\end{tabular}

GO, gene ontology; KEGG, kyoto encyclopedia of genes and genomes.

As a scaffolding protein, NEDD9, also termed human enhancer of filamentation $1(H E F l)$ is involved in mediating several cellular processes, including cell cycle progression, cellular attachment, apoptosis, motility and inflammation $(35,36)$. Prostaglandin E2 (PGE2) promotes the expression of $H E F 1$ and then induces cell cycle progression, and HEF1 is an important downstream mediator of the activity of $P G E 2$ in the progression of CRC (37). The activation and overexpression of extracellular signal-regulated kinase/MAPK is important in the progression of CRC and can be a molecular target for the treatment of the cancer (38). It has been reported that $M A P 2 K$ inhibitors can be of use in CRC therapy (39). Therefore, the dysregulation of NEDD9 and $M A P 2 K 2$ may affect CRC. In the PPI network of the top 50 nodes in the present study, CCND1 was also found to have interactions with BCAS1, NEDD9 and MAP2K2, indicating that CCND1 may also function in CRC through BCAS1, NEDD 9 and $M A P 2 K 2$.

In conclusion, the present study performed a comprehensive bioinformatics analysis of genes, which may be involved in CRC. A total of 1,640 DEGs were screened in the CRC samples, compared with normal samples. It was found that DEPTOR, AURKA,CCND1, BCAS1, NEDD9 and $M A P 2 K 2$ may be correlated with CRC. However, further confirmation is required to elucidate their mechanisms of action in CRC.

\section{References}

1. Kune GA, Kune S and Watson LF: Colorectal cancer risk, chronic illnesses, operations and medications: Case control results from the melbourne colorectal cancer study. Cancer Res 48: 4399-4404, 1988

2. Majumdar SR, Fletcher RH and Evans AT: How does colorectal cancer present?; symptoms, duration, and clues to location. Am J Gastroenterol 94: 3039-3045, 1999.
3. Brenner H, Bouvier AM, Foschi R, Hackl M, Larsen IK, Lemmens V, Mangone L and Francisci S: Progress in colorectal cancer survival in Europe from the late 1980 s to the early $21 \mathrm{st}$ century: The EUROCARE study. Int J Cancer 131: 1649-1658, 2012.

4. Ferlay J, Parkin DM and Steliarova-Foucher E: Estimates of cancer incidence and mortality in Europe in 2008. Eur J Cancer 46: 765-781, 2010.

5. Cianchi F, Cortesini C, Bechi P, Fantappiè O, Messerini L, Vannacci A, Sardi I, Baroni G, Boddi V, Mazzanti R and Masini E: Up-regulation of cyclooxygenase 2 gene expression correlates with tumor angiogenesis in human colorectal cancer. Gastroenterology 121: 1339-1347, 2001.

6. Ikenoue T, Kanai F, Hikiba Y, Obata T, Tanaka Y, Imamura J, Ohta M, Jazag A, Guleng B, Tateishi K, et al: Functional analysis of PIK3CA gene mutations in human colorectal cancer. Cancer Res 65: 4562-4567, 2005.

7. Smith G, Carey FA, Beattie J, Wilkie MJ, Lightfoot TJ, Coxhead J, Garner RC, Steele RJ and Wolf CR: Mutations in APC, Kirsten-ras, and p53-alternative genetic pathways to colorectal cancer. Proc Natl Acad Sci USA 99: 9433-9438, 2002.

8. Suzuki H, Watkins DN, Jair KW, Schuebel KE, Markowitz SD, Chen WD, Pretlow TP, Yang B, Akiyama Y, van Engeland M, et al: Epigenetic inactivation of SFRP genes allows constitutive WNT signaling in colorectal cancer. Nat Genet 36: 417-422, 2004

9. Cui H, Cruz-Correa M, Giardiello FM, Hutcheon DF, Kafonek DR, Brandenburg S, Wu Y, He X, Powe NR and Feinberg AP: Loss of IGF2 imprinting: A potential marker of colorectal cancer risk. Science 299: 1753-1755, 2003.

10. Khamas A, Ishikawa T, Shimokawa K, Mogushi K, Iida S, Ishiguro M, Mizushima H, Tanaka H, Uetake H and Sugihara K: Screening for epigenetically masked genes in colorectal cancer Using 5-Aza-2'-deoxycytidine, microarray and gene expression profile. Cancer Genomics-Proteomics 9: 67-75, 2012.

11. Gautier L, Cope L, Bolstad BM and Irizarry RA: affy-analysis of Affymetrix GeneChip data at the probe level. Bioinformatics 20: 307-315, 2004.

12. Smyth GK: Limma: Linear models for microarray data. In: Bioinformatics and computational biology solutions using $\mathrm{R}$ and bioconductor. edn: Springer pp397-420, 2005.

13. Hamosh A, Scott AF, Amberger JS, Bocchini CA and McKusick VA: Online Mendelian Inheritance in Man (OMIM), a knowledgebase of human genes and genetic disorders. Nucleic acids Res 33: D514-D517, 2005. 
14. Szklarczyk D, Franceschini A, Kuhn M, Simonovic M, Roth A Minguez P, Doerks T, Stark M, Muller J, Bork P, et al: The STRING database in 2011: Functional interaction networks of proteins, globally integrated and scored. Nucleic acids Res 39: D561-D568, 2011.

15. Fang $\mathrm{H}$ and Gough J: A disease-drug-phenotype matrix inferred by walking on a functional domain network. Mol Biosyst 9: 1686-1696, 2013

16. Fang $\mathrm{H}$ and Gough J: The 'dnet' approach promotes emerging research on cancer patient survival. Genome Med 6: 64, 2014.

17. Sherman BT, Huang da W, Tan Q, Guo Y, Bour S, Liu D, Stephens R, Baseler MW, Lane HC and Lempicki RA: DAVID Knowledgebase: A gene-centered database integrating heterogeneous gene annotation resources to facilitate high-throughput gene functional analysis. BMC Bioinformatics 8: 426, 2007.

18. Hulsegge I, Kommadath A and Smits MA: Globaltest and GOEAST: Two different approaches for Gene Ontology analysis. BMC Proc 3 (Suppl 4): S10, 2009.

19. Ogata H, Goto S, Sato K, Fujibuchi W, Bono H and Kanehisa M: KEGG: Kyoto encyclopedia of genes and genomes. Nucleic acids Res 27: 29-34, 1999.

20. Griffith M, Griffith OL, Coffman AC, Weible JV, McMichael JF, Spies NC, Koval J, Das I, Callaway MB, Eldred JM, et al: DGIdb: Mining the druggable genome. Nat Methods 10: 1209-1210, 2013.

21. Lail EY, Chen ZG, Zhou X, Fan XR, Wang H, Lai PL, Su YC, Zhang BY, Bai XC and Li YF: DEPTOR Expression negatively correlates with mTORC1 activity and tumor progression in colorectal cancer. Asian Pac J Cancer Prev 15: 4589-4594, 2014

22. Francipane MG and Lagasse E: mTOR pathway in colorectal cancer: An update. Oncotarget 5: 49, 2014.

23. Grünhage F, Jungck M, Lamberti C, Berg C, Becker U, Schulte-Witte H, Plassmann D, Rahner N, Aretz S, Friedrichs N, et al: Association of familial colorectal cancer with variants in the E-cadherin (CDH1) and cyclin D1 (CCND1) genes. Int J Colorectal Dis 23: 147-154, 2008.

24. Lewis RC, Bostick RM, Xie D, Deng Z, Wargovich MJ, Fina MF, Roufail WM and Geisinger KR: Polymorphism of the cyclin D1 gene, CCND1, and risk for incident sporadic colorectal adenomas. Cancer Res 63: 8549-8553, 2003.

25. Zhang W, Gordon M, Press OA, Rhodes K, Vallböhmer D, Yang DY, Park D, Fazzone W, Schultheis A, Sherrod AE, et al: Cyclin D1 and epidermal growth factor polymorphisms associated with survival in patients with advanced colorectal cancer treated with Cetuximab. Pharmacogenet Genomics 16: 475-483, 2006.

26. Goos JA, Coupe VM, Diosdado B, Delis-Van Diemen PM, Karga C, Beliën JA, Carvalho B, van den Tol MP, Verheul HM, Geldof AA, et al: Aurora kinase A (AURKA) expression in colorectal cancer liver metastasis is associated with poor prognosis. Br J Cancer 109: 2445-2452, 2013.
27. Bischoff JR, Anderson L, Zhu Y, Mossie K, Ng L, Souza B, Schryver B, Flanagan P, Clairvoyant F, Ginther C, et al: A homologue of Drosophila aurora kinase is oncogenic and amplified in human colorectal cancers. EMBO J 17: 3052-3065, 1998.

28. Hermsen M, Postma C, Baak J, Weiss M, Rapallo A, Sciutto A Roemen G, Arends JW, Williams R, Giaretti W, et al: Colorectal adenoma to carcinoma progression follows multiple pathways of chromosomal instability. Gastroenterology 123: 1109-1119, 2002.

29. Aust DE, Muders M, Köhler A, Schmidt M, Diebold J, Müller C, Löhrs U, Waldman FM and Baretton GB: Prognostic relevance of 20q13 gains in sporadic colorectal cancers: A FISH analysis. Scand J Gastroenterol 39: 766-772, 2004.

30. Nakao K, Mehta KR, Fridlyand J, Moore DH, Jain AN, Lafuente A, Wiencke JW, Terdiman JP and Waldman FM: High-resolution analysis of DNA copy number alterations in colorectal cancer by array-based comparative genomic hybridization. Carcinogenesis 25: 1345-1357, 2004.

31. Postma C, Terwischa S, Hermsen MA, Van der Sijp JR and Meijer GA: Gain of chromosome $20 \mathrm{q}$ is an indicator of poor prognosis in colorectal cancer. Cell Oncol 29: 73-75, 2007.

32. Carvalho B, Postma C, Mongera S, Hopmans E, Diskin S, van de Wiel MA, Van Criekinge W, Thas O, Matthäi A, Cuesta MA, et al: Multiple putative oncogenes at the chromosome 20q amplicon contribute to colorectal adenoma to carcinoma progression. Gut 58: 79-89, 2009.

33. Sillars-Hardebol AH, Carvalho B, de Wit M, Postma C, Delis-van Diemen PM, Mongera S, Ylstra B, van de Wiel MA, Meijer GA and Fijneman RJ: Identification of key genes for carcinogenic pathways associated with colorectal adenoma-to-carcinoma progression. Tumour Biol 31: 89-96, 2010.

34. Correa RG, de Carvalho AF, Pinheiro NA, Simpson AJ and De Souza SJ: NABC1 (BCAS1): Alternative splicing and downregulation in colorectal tumors. Genomics 65: 299-302, 2000.

35. Kim SH, Xia D, Kim SW, Holla V, Menter DG and DuBois RN: Human enhancer of filamentation 1 is a mediator of hypoxia-inducible factor-1alpha-mediated migration in colorectal carcinoma cells. Cancer Res 70: 4054-4063, 2010.

36. Singh MK, Cowell L, Seo S, O'Neill G and Golemis E: Molecular basis for HEF1/NEDD9/Cas-L action as a multifunctional co-ordinator of invasion, apoptosis and cell cycle. Cell Biochem Biophys 48: 54-72, 2007.

37. Xia D, Holla VR, Wang D, Menter DG and DuBois RN: HEF1 is a crucial mediator of the proliferative effects of prostaglandin $\mathrm{E}(2)$ on colon cancer cells. Cancer Res 70: 824-831, 2010.

38. Fang JY and Richardson BC: The MAPK signalling pathways and colorectal cancer. Lancet Oncol 6: 322-327, 2005.

39. Yeh JJ, Routh ED, Rubinas T, Peacock J, Martin TD, Shen XJ, Sandler RS, Kim HJ, Keku TO and Der CJ: KRAS/BRAF mutation status and ERK1/2 activation as biomarkers for MEK1/2 inhibitor therapy in colorectal cancer. Mol Cancer Ther 8: 834-843, 2009. 